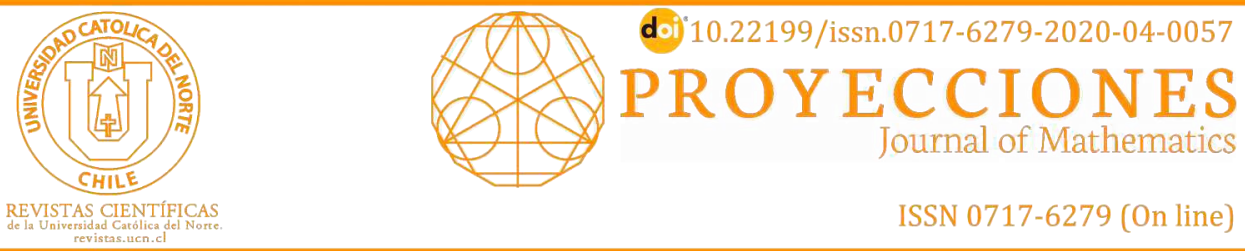

\title{
Some resistance distance and distance-based graph invariants and number of spanning trees in the tensor product of $P_{2}$ and $K_{n}$
}

\author{
Muhammad Shoaib Sardar ${ }^{1}$ (b) orcid.org/0000-0001-7146-5639 \\ Murat Cancan ${ }^{2}$ (으 orcid.org/0000-0002-8606-2274 \\ Süleyman Ediz ${ }^{3}$ (1) orcid.org/0000-0003-0625-3634 \\ Wasim Sajjad ${ }^{4}$ 응 orcid.org/0000-0002-2695-0237 \\ ${ }^{1}$ Anhui University, School of Mathematical Sciences, Hefei, Anhui, China. \\ shoaibsardar093@gmail.com \\ ${ }^{2}$ Van Yüzüncü Yil University, Faculty of Education, Van, Turkey. \\ m_cencen@yahoo.com \\ ${ }^{3}$ Van Yüzüncü Yil University, Faculty of Education, Van, Turkey. \\ suleymanediz@yyu.edu.tr \\ ${ }^{4}$ Anhui University, School of Mathematical Sciences, Hefei, Anhui, China. \\ wasim.sajjad89@gmail.com
}

Received: February 2020 | Accepted: May 2020

\section{Abstract:}

The resistance distance (Kirchhoff index and multiplicative Kirchhoff index) and distance-based (Wiener index and Gutman index) graph invariants of $\Gamma_{n}=P_{2} \times K_{n}$ are considered. Firstly by using the decomposition theorem, we procure the Laplacian and Normalized Laplacian spectrum for graph $\Gamma_{n}$, respectively. Based on which, we can procured the formulae for the number of spanning trees and some resistance distance and distancebased graph invariants of graph $\Gamma_{n}$. Also, it is very interesting to see that when $n$ tends to infinity, $K f\left(\Gamma_{n}\right)$ is a polynomial and $W\left(\Gamma_{n}\right)$ is a quadratic polynomial.

Keywords: Kirchhoff index; Degree-Kirchhoff index; Normalized Laplacian; Spanning tree.

MSC (2020): 05C09.

\section{Cite this article as (IEEE citation style)}

M. S. Sardar, M. Cancan, S. Ediz, and W. Sajjad., "Some resistance distance and distance-based graph invariants and number of spanning trees in the tensor product of $P_{2}$ and $K_{n}^{\prime \prime}$, Proyecciones (Antofagasta, On line), vol. 39, no. 4, pp. 919-932, Aug. 2020, doi: $10.22199 /$ issn.0717-6279-2020-04-0057.

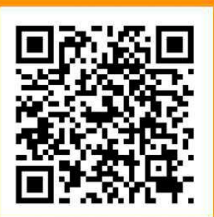

Article copyright: (C) 2020 Muhammad Shoaib Sardar, Murat Cancan, Süleyman Ediz and Wasim Sajjad. . This is an open access article distributed under the terms of the Creative Commons License, which permits unrestricted use and distribution provided the original author and source are credited. 


\section{Introduction}

The graphs studied in the present paper are undirected and connected. For undefined notations and terminologies discussed in this paper but not defined here, see the book by Bondy and Murty [3]. Resistance distance is an intrinsic graph metric and a distance function on graphs which is further extensions of ordinary distance. Let $\mathcal{G}$ and $\mathcal{H}$ be two graphs. The tensor product $\mathcal{G} \times \mathcal{H}$ of $\mathcal{G}$ and $\mathcal{H}$ is a graph whose vertex set is the Cartesian product of $V(\mathcal{G}) \times V(\mathcal{H})$ and distinct vertices $\left(a, a^{\prime}\right)$ and $\left(b, b^{\prime}\right)$ are adjacent in $\mathcal{G} \times \mathcal{H}$ if $a$ is adjacent to $b$ and $a^{\prime}$ is adjacent to $b^{\prime}$.

Suppose $\Gamma=\left(V_{\Gamma}, E_{\Gamma}\right)$ is a graph with vertex-set $V_{\Gamma}$ and edge-set $E_{\Gamma}$. The adjacency matrix $A(\Gamma)$ of a graph $\Gamma$ is a $\left|V_{\Gamma} \times V_{\Gamma}\right|$ matrix whose $(i, j)$-entry equals to 1 if vertices $v_{i}$ and $v_{j}$ are adjacent and 0 otherwise. Suppose $D(\Gamma)=\left(d_{1}, d_{2}, \ldots, d_{n}\right)$ is a diagonal matrix of a graph $\Gamma$, where $d_{1}, d_{2}, \ldots, d_{n}$ are the degree of vertices $\left(v_{1}, v_{2}, \ldots, v_{n}\right)$. Suppose $Q(\Gamma)=D(\Gamma)+A(\Gamma)$ is a Signless Laplacian matrix of a graph $\Gamma$.

The Laplacian matrix for a graph $\Gamma$ is determined as

$$
L(\Gamma)_{i j}= \begin{cases}d_{i}, & \text { if } i \text { is equal to } j \\ -1, & \text { if } i \text { is not equal to } j \text { and } v_{i} \sim v_{j} \\ 0, & \text { otherwise. }\end{cases}
$$

The Normalized Laplacian matrix for a graph $\Gamma$ is determined as

$$
\mathcal{L}(\Gamma)_{i j}= \begin{cases}1, & \text { if } i \text { is equal to } j \\ -\frac{1}{\sqrt{d_{i} d_{j}}}, & \text { if } i \text { is not equal to } j \text { and } v_{i} \sim v_{j} \\ 0, & \text { otherwise. }\end{cases}
$$

Clearly, $L(\Gamma)=D(\Gamma)-A(\Gamma)$ and $\mathcal{L}(\Gamma)=D(\Gamma)^{\frac{-1}{2}} L(\Gamma) D(\Gamma)^{\frac{-1}{2}}$. The distance among two vertices $i$ and $j$ is the length of shortest walk among vertices $i$ and $j$ and is represented by $d_{i j}$, which is very important in graph theory [4]. There are number of parameters to characterize the structural properties of graphs. The Wiener index [24] is determined in the following way:

$$
W(\Gamma)=\sum_{i<j} d_{i j}
$$


Later, the another version of Wiener index was presented by Gutman which is known by his name called Gutman index [11] and determined in the following way:

$$
G u t(\Gamma)=\sum_{i<j} d_{i} d_{j} d_{i j} .
$$

In 1993, Klein and Randić[17] initiated the study of resistance distance based on electrical network. The resistance distance, denoted by $r(u, v)$, is described as the net effective resistance among vertices $u$ and $v$ of $G$ in the corresponding electrical network obtained from $G$ by changing each edge of $G$ with a resistor of $1 \mathrm{Ohm}$. They also presented the Krichhoff index for a graph $\Gamma$ which is determined in the following way:

$$
K f(\Gamma)=\sum_{i<j} r_{i j} .
$$

For a connected graph $(\Gamma)$, the Kirchhoff index with regard to Laplacian matrix is also proved by Zhu et al. [28] and Gutman and Mohar [12] and determined in the following way:

$$
K f(\Gamma)=n \sum_{i=2}^{n} \frac{1}{\omega_{i}},
$$

where $0=\omega_{1}<\omega_{2} \leq \ldots \leq \omega_{n}$ are the eigenvalues of $L(\Gamma)$.

In 2007, the weighted version of Kirchhoff index was suggested in [6], determined in the following way:

$$
K f^{*}(\Gamma)=\sum_{i<j} d_{i} d_{j} r_{i j} .
$$

This index is called multiplicative Kirchhoff index. It is also relevant to spectrum of the Normalized Laplacian matrix and it is also determined in the following way $[6]$ :

$$
K f^{*}(\Gamma)=2 m \sum_{i=2}^{n} \frac{1}{\psi_{i}},
$$

where $m=\left|E_{\Gamma}\right|, 0=\psi_{1}<\psi_{2} \leq \ldots \leq \psi_{n}$ represents the eigenvalues of $\mathcal{L}(\Gamma)$.

Kirchhoff index is also a graph structure-descriptor [25] similar to Wiener index. In spite of, it is not simple to get a closed formula for Kirchhoff index due to its computational complexity but there are different kinds of 
techniques to determine the Kirchhoff index (see $[1,2,9,10,29]$ ). In recent times, Kirchhoff index and multiplicative Kirchhoff index were computed for different graph structures, i.e., composite graphs [27], ladder graphs [8], Cycles [16], ladder-like chains [5], linear polyomino chains [15,26], linear octagonal-quadrilateral networks [18], linear pentagonal chains [13], linear phenylenes [19,29] and linear hexagonal chains [21, 22].

Suppose $\mathcal{G}$ and $\mathcal{H}$ are two graphs. The tensor product of $\mathcal{G}$ and $\mathcal{H}$ is a graph whose vertex set is the Cartesian product of $V(\mathcal{G}) \times V(\mathcal{H})$ and distinct vertices $\left(a, a^{\prime}\right)$ and $\left(b, b^{\prime}\right)$ are adjacent in $\mathcal{G} \times \mathcal{H}$ if $a$ is adjacent to $b$ and $a^{\prime}$ is adjacent to $b^{\prime}$ and it is represented by $\mathcal{G} \times \mathcal{H}$. Let $\Gamma_{n}=P_{2} \times K_{n}$ be a graph obtained by tensor product of $P_{2}$ and $K_{n}$. Clearly, the graph $\Gamma_{n}$ contain $2 n$ vertices and $n(n-1)$ edges. The graph illustrated in Figure 1.1 is an example of tensor product of path graph $P_{2}$ and a complete graph $K_{4}$.

The present study is organized in this way. In Section 2, there are few elementary definitions that leads to main results in Section 3. The conclusive remarks for the whole paper are discussed in Section 4.

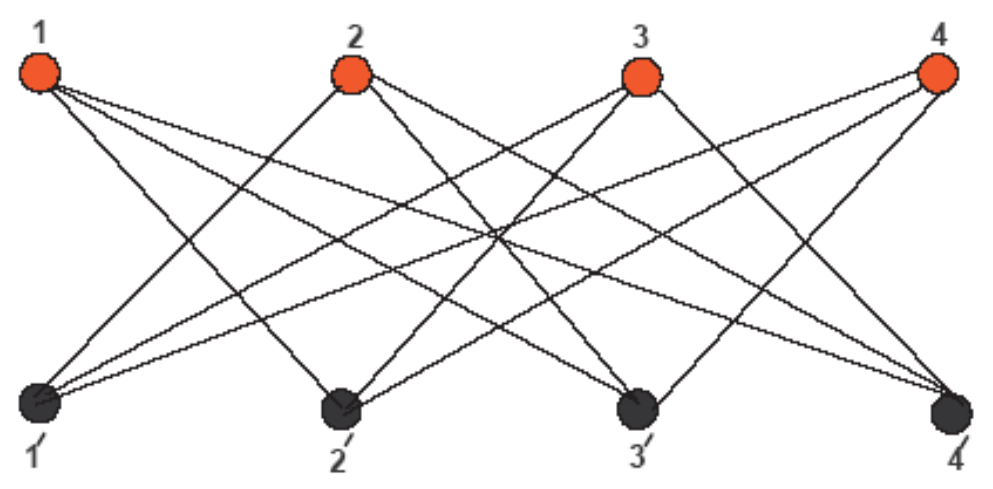

Figure 1.1: The graph $\Gamma_{4}=P_{2} \times K_{4}$. 


\section{Preliminaries}

Suppose $M$ is a square matrix and the characteristic polynomial for $M$ is $\varphi(M)=\operatorname{det}(x I-M)$. Label the vertices of $\Gamma_{n}$ like Figure 1.1. Let $V_{1}=\{1,2, \ldots, n\}$ and $V_{2}=\left\{1^{\prime}, 2^{\prime}, \ldots, n^{\prime}\right\}$. It is easy to see that there is an automorphism i.e., $\pi=\left(1,1^{\prime}\right),\left(2,2^{\prime}\right), \ldots,\left(n, n^{\prime}\right)$. Then we can write the Laplacian matrix and Normalized Laplacian matrix of $\Gamma_{n}$ in the following way.

$$
L\left(\Gamma_{n}\right)=\left(\begin{array}{ll}
L_{V_{11}} & L_{V_{12}} \\
L_{V_{21}} & L_{V_{22}}
\end{array}\right), \mathcal{L}\left(\Gamma_{n}\right)=\left(\begin{array}{cc}
\mathcal{L}_{V_{11}} & \mathcal{L}_{V_{12}} \\
\mathcal{L}_{V_{21}} & \mathcal{L}_{V_{22}}
\end{array}\right)
$$

It is easy to check that $L_{V_{11}}=L_{V_{22}}, L_{V_{12}}=L_{V_{21}}$ and $\mathcal{L}_{V_{11}}=\mathcal{L}_{V_{22}}$, $\mathcal{L}_{V_{12}}=\mathcal{L}_{V_{21}}$.

Given

$$
T=\left(\begin{array}{cc}
\frac{1}{\sqrt{2}} I_{n} & \frac{1}{\sqrt{2}} I_{n} \\
\frac{1}{\sqrt{2}} I_{n} & -\frac{1}{\sqrt{2}} I_{n}
\end{array}\right)
$$

then

$T L\left(\Gamma_{n}\right) T^{\prime}=\left(\begin{array}{cc}L_{A} & 0 \\ 0 & L_{S}\end{array}\right)$ and $T \mathcal{L}\left(\Gamma_{n}\right) T^{\prime}=\left(\begin{array}{cc}\mathcal{L}_{A} & 0 \\ 0 & \mathcal{L}_{S}\end{array}\right)$

where $T^{\prime}$ is the transposition of $T, L_{A}=L_{V_{11}}+L_{V_{12}}, L_{S}=L_{V_{11}}-L_{V_{12}}$ and $\mathcal{L}_{A}=\mathcal{L}_{V_{11}}+\mathcal{L}_{V_{12}}, \mathcal{L}_{S}=\mathcal{L}_{V_{11}}-\mathcal{L}_{V_{12}}$.

We can procure the decomposition theorem of graph $\Gamma_{n}$ as follows.

Lemma[14,21] Suppose $L_{A}, L_{S}, \mathcal{L}_{A}$ and $\mathcal{L}_{S}$ are matrices as defined above. Then $\varphi\left(L\left(\Gamma_{n}\right)\right)=\varphi\left(L_{A}\right) \cdot \varphi\left(L_{S}\right)$ and $\varphi\left(\mathcal{L}\left(\Gamma_{n}\right)\right)=\varphi\left(\mathcal{L}_{A}\right) \cdot \varphi\left(\mathcal{L}_{S}\right)$.

Lemma[7] Suppose $\Gamma$ is a $n$-vertex connected graph and $\tau(\Gamma)$ represents the number of spanning trees for a graph $\Gamma$. Then

$$
\tau(\Gamma)=\frac{1}{n} \prod_{i=2}^{n} \omega_{i}
$$

where $0=\omega_{1}<\omega_{2} \leq \ldots \leq \omega_{n}$ are the eigenvalues of $L(\Gamma)$.

\section{The main results}

In the present section, we propose the explicit formulas for the Kirchhoff index, Wiener index, multiplicative Kirchhoff index, Gutman index and 
number of spanning trees for $\Gamma_{n}$. First, one can see that

$$
=\left(\begin{array}{ccccccc}
n-1 & 0 & 0 & 0 & \cdots & 0 & 0 \\
0 & n-1 & 0 & 0 & \cdots & 0 & 0 \\
0 & 0 & n-1 & 0 & \cdots & 0 & 0 \\
0 & 0 & 0 & n-1 & \cdots & 0 & 0 \\
\vdots & \vdots & \vdots & \vdots & \ddots & \vdots & \vdots \\
0 & 0 & 0 & 0 & \cdots & n-1 & 0 \\
0 & 0 & 0 & 0 & \cdots & 0 & n-1
\end{array}\right)_{n \times n},
$$

where $\ell_{i, i}=n-1$ for $i \in\{1,2, \ldots, n\}$ and all other entries of $L_{V_{11}}=\left(\ell_{i j}\right)_{n \times n}^{V_{11}}$ are equal to zero.

$$
\begin{gathered}
L_{V_{12}}=\left(\ell_{i j}\right)_{n \times n}^{V_{12}} \\
=\left(\begin{array}{ccccccc}
0 & -1 & -1 & -1 & \cdots & -1 & -1 \\
-1 & 0 & -1 & -1 & \cdots & -1 & -1 \\
-1 & -1 & 0 & -1 & \cdots & -1 & -1 \\
-1 & -1 & -1 & 0 & \cdots & -1 & -1 \\
\vdots & \vdots & \vdots & \vdots & \ddots & \vdots & \vdots \\
-1 & -1 & -1 & -1 & \cdots & 0 & -1 \\
-1 & -1 & -1 & -1 & \cdots & -1 & 0
\end{array}\right)_{n \times n},
\end{gathered}
$$

where $\ell_{i, i}=0$ for $i \in\{1,2, \ldots, n\}$ and and all other entries of $L_{V_{12}}=$ $\left(\ell_{i j}\right)_{n \times n}^{V_{12}}$ are equal to -1 . Since $L_{A}=L_{V_{11}}+L_{V_{12}}$ and $L_{S}=L_{V_{11}}-L_{V_{12}}$, then

$$
=\left(\begin{array}{ccccccc}
n-1 & -1 & -1 & -1 & \cdots & -1 & -1 \\
-1 & n-1 & -1 & -1 & \cdots & -1 & -1 \\
-1 & -1 & n-1 & -1 & \cdots & -1 & -1 \\
-1 & -1 & -1 & n-1 & \cdots & -1 & -1 \\
\vdots & \vdots & \vdots & \vdots & \ddots & \vdots & \vdots \\
-1 & -1 & -1 & -1 & \cdots & n-1 & -1 \\
-1 & -1 & -1 & -1 & \cdots & -1 & n-1
\end{array}\right)_{n \times n}
$$

where $\ell_{i, i}=n-1$ for $i \in\{1,2, \ldots, n\}$ and all other entries of $L_{A}=\left(\ell_{i j}\right)_{n \times n}^{V_{A}}$ are equal to -1 . Now

$$
L_{S}=\left(\ell_{i j}\right)_{n \times n}^{V_{S}}
$$




$$
=\left(\begin{array}{ccccccc}
n-1 & 1 & 1 & 1 & \cdots & 1 & 1 \\
1 & n-1 & 1 & 1 & \cdots & 1 & 1 \\
1 & 1 & n-1 & 1 & \cdots & 1 & 1 \\
1 & 1 & 1 & n-1 & \cdots & 1 & 1 \\
\vdots & \vdots & \vdots & \vdots & \ddots & \vdots & \vdots \\
1 & 1 & 1 & 1 & \cdots & n-1 & 1 \\
1 & 1 & 1 & 1 & \cdots & 1 & n-1
\end{array}\right)_{n \times n}
$$

where $\ell_{i, i}=n-1$ for $i \in\{1,2, \ldots, n\}$ and all other entries of $L_{S}=\left(\ell_{i j}\right)_{n \times n}^{V_{S}}$ are equal to 1 .

Note that $L_{A}=L\left(K_{n}\right)$ and $L_{S}=Q\left(K_{n}\right)$, where $L_{A}$ is equal to Laplacian matrix of a complete graph $K_{n}$ and $L_{S}$ is equal to Signless Laplacian matrix of a complete graph $K_{n}$. It is renowned that eigenvalues of Laplacian matrix of complete graph $K_{n}$ are $n$ with multiplicity $n-1$ and 0 with multiplicity 1 and the eigenvalues of Signless laplacian matrix of complete graph $K_{n}$ are $n-2$ with multiplicity $n-1$ and $2 n-2$ with the multiplicity 1 . By using Lemma $1, \phi_{1}, \phi_{2}, \ldots, \phi_{n}, \eta_{1}, \eta_{2}, \ldots, \eta_{n}$ represents the eigenvalues of $L\left(\Gamma_{n}\right)$, i.e., $0, n, n, \ldots, n, n-2, n-2, \ldots, n-2,2 n-2$ are all the eigenvalues of $L\left(\Gamma_{n}\right)$.

Theorem 3.1 Let $\Gamma_{n}(n \geq 3)$ be a graph as defined above. Then

1. $K f\left(\Gamma_{n}\right)=\frac{4 n^{3}-11 n^{2}+10 n-4}{n^{2}-3 n+2}$.

2. $\tau\left(\Gamma_{n}\right)=n^{n-2}(n-1)(n-2)^{n-1}$.

3. $W\left(\Gamma_{n}\right)=3 n^{2}$.

4. $\lim _{n \rightarrow+\infty} \frac{K f\left(\Gamma_{n}\right)}{W\left(\Gamma_{n}\right)}=0$

Proof. (1) Since $\left|V\left(\Gamma_{n}\right)\right|=2 n$. So by using Equation 1.3, we procure

$$
\begin{aligned}
K f\left(\Gamma_{n}\right) & =2 n\left(\sum_{i=2}^{n} \frac{1}{\phi_{i}}+\sum_{i=1}^{n} \frac{1}{\eta_{i}}\right) \\
& =2 n\left(\frac{n-1}{n}+\frac{1}{2 n-2}+\frac{n-1}{n-2}\right) \\
& =n\left(\frac{4 n^{3}-11 n^{2}+10 n-4}{n(n-1)(n-2)}\right) \\
& =\frac{4 n^{3}-11 n^{2}+10 n-4}{n^{2}-3 n+2} .
\end{aligned}
$$

(2) Since $\left|V\left(\Gamma_{n}\right)\right|=2 n$. So by using Lemma 2, we obtain 


$$
\begin{aligned}
\tau\left(\Gamma_{n}\right) & =\frac{1}{2 n}\left(\prod_{i=2}^{n} \phi_{i} \cdot \prod_{i=1}^{n} \eta_{i}\right) \\
& =\frac{1}{2 n}\left((n)^{n-1} \cdot(2 n-2) \cdot(n-2)^{n-1}\right) \\
& =n^{n-2}(n-1)(n-2)^{n-1} .
\end{aligned}
$$

(3) Now we determine the Wiener index for graph $\Gamma_{n}$.

Since the diameter of graph $\Gamma_{n}$ is 3 . So we have three different pairs of vertices i.e., $n(n-1), \sum_{i=1}^{n} n-1=n(n-1)$ and $n$ pairs of vertices at a distance 1, 2 and 3, respectively. So by using equation 1.1, it is easy to obtain the desired result, i.e.,

$$
W\left(\Gamma_{n}\right)=n(n-1)+2 n(n-1)+3 n=3 n^{2} .
$$

Table 3.1: $K f(\Gamma)$ (reserves a decimal fraction) and $\tau(\Gamma)$ of graphs from $\Gamma_{4}$ to $\Gamma_{12}$.

\begin{tabular}{ccccccccc}
\hline$\Gamma$ & $K f(\Gamma)$ & $\tau(\Gamma)$ & $\Gamma$ & $K f(\Gamma)$ & $\tau(\Gamma)$ & $\Gamma$ & $K f(\Gamma)$ & $\tau(\Gamma)$ \\
\hline$\Gamma_{4}$ & 19.3 & 384 & $\Gamma_{7}$ & 30 & 1575656250 & $\Gamma_{10}$ & 41.6 & 120795955200000000 \\
$\Gamma_{5}$ & 22.6 & 40500 & $\Gamma_{8}$ & 33.8 & 513684799488 & $\Gamma_{11}$ & 45.5 & 82216552273527685120 \\
$\Gamma_{6}$ & 26.2 & 6635520 & $\Gamma_{9}$ & 37.7 & 220582915793352 & $\Gamma_{12}$ & 49.5 & 68109100646400000000000 \\
\hline
\end{tabular}

(4) Combining with $K f\left(\Gamma_{n}\right)$ and $W\left(\Gamma_{n}\right)$, one gets

$$
\begin{aligned}
\lim _{n \rightarrow+\infty} \frac{K f\left(\Gamma_{n}\right)}{W\left(\Gamma_{n}\right)} & =\lim _{n \rightarrow+\infty} \frac{4 n^{3}-11 n^{2}+10 n-4}{3 n^{4}-9 n^{3}+6 n^{2}} \\
& =\lim _{n \rightarrow+\infty} \frac{4-11 \frac{1}{n}+10 \frac{1}{n^{2}}-4 \frac{1}{n^{3}}}{3 n-9+6 \frac{1}{n}} \\
& =0
\end{aligned}
$$

Now we determine the Normalized Laplacian spectrum for graph $\Gamma_{n}$. Note that

$$
\mathcal{L}_{V_{11}}=\left(\ell_{i j}\right)_{n \times n}^{V_{11}}
$$




$$
=\left(\begin{array}{ccccccc}
1 & 0 & 0 & 0 & \cdots & 0 & 0 \\
0 & 1 & 0 & 0 & \cdots & 0 & 0 \\
0 & 0 & 1 & 0 & \cdots & 0 & 0 \\
0 & 0 & 0 & 1 & \cdots & 0 & 0 \\
\vdots & \vdots & \vdots & \vdots & \ddots & \vdots & \vdots \\
0 & 0 & 0 & 0 & \cdots & 1 & 0 \\
0 & 0 & 0 & 0 & \cdots & 0 & 1
\end{array}\right)_{n \times n}
$$

where $\ell_{i, i}=1$ for $i \in\{1,2, \ldots, n\}$ and all other entries of $\mathcal{L}_{V_{11}}=\left(\ell_{i j}\right)_{n \times n}^{V_{11}}$ are equal to zero.

Now

$$
\begin{gathered}
L_{V_{12}}=\left(\ell_{i j}\right)_{2 n \times 2 n}^{V_{12}} \\
=\left(\begin{array}{ccccccc}
0 & \frac{-1}{n-1} & \frac{-1}{n-1} & \frac{-1}{n-1} & \cdots & \frac{-1}{n-1} & \frac{-1}{n-1} \\
\frac{-1}{n-1} & 0 & \frac{-1}{n-1} & \frac{-1}{n-1} & \cdots & \frac{-1}{n-1} & \frac{-1}{n-1} \\
\frac{-1}{n-1} & \frac{-1}{n-1} & 0 & \frac{-1}{n-1} & \cdots & \frac{-1}{n-1} & \frac{-1}{n-1} \\
\frac{-1}{n-1} & \frac{-1}{n-1} & \frac{-1}{n-1} & 0 & \cdots & \frac{-1}{n-1} & \frac{-1}{n-1} \\
\vdots & \vdots & \vdots & \vdots & \ddots & \vdots & \vdots \\
\frac{-1}{n-1} & \frac{-1}{n-1} & \frac{-1}{n-1} & \frac{-1}{n-1} & \cdots & 0 & -\frac{-1}{n-1} \\
\frac{-1}{n-1} & \frac{-1}{n-1} & \frac{-1}{n-1} & \frac{-1}{n-1} & \cdots & \frac{-1}{n-1} & 0
\end{array}\right)_{n \times n},
\end{gathered}
$$

where $\ell_{i, i}=0$ for $i \in\{1,2, \ldots, n\}$ and all other entries of $\mathcal{L}_{V_{12}}=\left(\ell_{i j}\right)_{n \times n}^{V_{12}}$ are equal to $-\frac{1}{n-1}$. Since $\mathcal{L}_{A}=\mathcal{L}_{V_{11}}+\mathcal{L}_{V_{12}}$ and $\mathcal{L}_{S}=\mathcal{L}_{V_{11}}-\mathcal{L}_{V_{12}}$, then we have

$$
\begin{gathered}
\mathcal{L}_{A}=\left(\ell_{i j}\right)_{n \times n}^{V_{A}} \\
=\left(\begin{array}{ccccccc}
1 & \frac{-1}{n-1} & \frac{-1}{n-1} & \frac{-1}{n-1} & \cdots & \frac{-1}{n-1} & \frac{-1}{n-1} \\
\frac{-1}{n-1} & 1 & \frac{-1}{n-1} & \frac{-1}{n-1} & \cdots & \frac{-1}{n-1} & \frac{-1}{n-1} \\
\frac{-1}{n-1} & \frac{-1}{n-1} & 1 & \frac{-1}{n-1} & \cdots & \frac{-1}{n-1} & \frac{-1}{n-1} \\
\frac{-1}{n-1} & \frac{-1}{n-1} & \frac{-1}{n-1} & 1 & \cdots & \frac{-1}{n-1} & \frac{-1}{n-1} \\
\vdots & \vdots & \vdots & \vdots & \ddots & \vdots & \vdots \\
\frac{-1}{n-1} & \frac{-1}{n-1} & \frac{-1}{n-1} & \frac{-1}{n-1} & \cdots & 1 & \frac{-1}{n-1} \\
\frac{-1}{n-1} & \frac{-1}{n-1} & \frac{-1}{n-1} & \frac{-1}{n-1} & \cdots & \frac{-1}{n-1} & 1
\end{array}\right)_{n \times n},
\end{gathered}
$$

where $\ell_{i, i}=1$ for $i \in\{1,2, \ldots, n\}$ and all other entries of $\mathcal{L}_{A}=\left(\ell_{i j}\right)_{n \times n}^{V_{A}}$ are equal to $\frac{-1}{n-1}$. Now

$$
\mathcal{L}_{S}=\left(\ell_{i j}\right)_{n \times n}^{V_{S}}
$$




$$
=\left(\begin{array}{ccccccc}
1 & \frac{1}{n-1} & \frac{1}{n-1} & \frac{1}{n-1} & \cdots & \frac{1}{n-1} & \frac{1}{n-1} \\
\frac{1}{n-1} & 1 & \frac{1}{n-1} & \frac{1}{n-1} & \cdots & \frac{1}{n-1} & \frac{1}{n-1} \\
\frac{1}{n-1} & \frac{1}{n-1} & 1 & \frac{1}{n-1} & \cdots & \frac{1}{n-1} & \frac{1}{n-1} \\
\frac{1}{n-1} & \frac{1}{n-1} & \frac{1}{n-1} & 1 & \cdots & \frac{1}{n-1} & \frac{1}{n-1} \\
\vdots & \vdots & \vdots & \vdots & \ddots & \vdots & \vdots \\
\frac{1}{n-1} & \frac{1}{n-1} & \frac{1}{n-1} & \frac{1}{n-1} & \cdots & 1 & \frac{1}{n-1} \\
\frac{1}{n-1} & \frac{1}{n-1} & \frac{1}{n-1} & \frac{1}{n-1} & \cdots & \frac{1}{n-1} & 1
\end{array}\right)_{n \times n}
$$

where $\ell_{i, i}=1$ for $i \in\{1,2, \ldots, n\}$ and all other entries of $\mathcal{L}_{S}=\left(\ell_{i j}\right)_{n \times n}^{V_{S}}$ are equal to $\frac{1}{n-1}$.

Also $\mathcal{L}_{A}=\frac{1}{n-1} L\left(K_{n}\right)$ and $\mathcal{L}_{S}=\frac{1}{n-1} Q\left(K_{n}\right)$. By using Lemma 1, $\frac{1}{n-1} \phi_{1}, \frac{1}{n-1} \phi_{2}, \ldots, \frac{1}{n-1} \phi_{n}, \frac{1}{n-1} \eta_{1}, \frac{1}{n-1} \eta_{2}, \ldots, \frac{1}{n-1} \eta_{n}$ are the eigenvalues of $L\left(\Gamma_{n}\right)$, i.e., $0, \frac{n}{n-1}, \frac{n}{n-1}, \ldots, \frac{n}{n-1}, \frac{n-2}{n-1}, \frac{n-2}{n-1}$,

$\ldots, \frac{n-2}{n-1}, \frac{2 n-2}{n-1}$ are all the eigenvalues of $\mathcal{L}\left(\Gamma_{n}\right)$.

Theorem 3.2 Suppose $\Gamma_{n}(n \geq 3)$ is a graph as defined above. Then

1. $K f^{*}\left(\Gamma_{n}\right)=(n-1)^{2} K f\left(\Gamma_{n}\right)=(n-1)\left[\frac{4 n^{3}-11 n^{2}+10 n-4}{n-2}\right]$.

2. $\operatorname{Gut}\left(\Gamma_{n}\right)=(n-1)^{2} W\left(\Gamma_{n}\right)=3 n^{2}(n-1)^{2}$.

3. $\lim _{n \rightarrow+\infty} \frac{K f^{*}\left(\Gamma_{n}\right)}{\operatorname{Gut}\left(\Gamma_{n}\right)}=0$

Proof. (1) Since $E\left(\Gamma_{n}\right)=n(n-1)$. So by using Equation 1.4, we have

$$
\begin{aligned}
K f^{*}\left(\Gamma_{n}\right) & =2 n(n-1)\left[\sum_{i=2}^{n} \frac{n-1}{\phi_{i}}+\sum_{i=1}^{n} \frac{n-1}{\eta_{i}}\right] \\
& =2 n(n-1)\left[\frac{(n-1)^{2}}{n}+\frac{n-1}{(2 n-2)}+\frac{(n-1)^{2}}{(n-2)}\right] \\
& =(n-1)\left[\frac{4 n^{3}-11 n^{2}+10 n-4}{n-2}\right] \\
& =(n-1)^{2} K f\left(\Gamma_{n}\right) .
\end{aligned}
$$

(2) Since $\Gamma_{n}$ is $(n-1)$ regular graph. So by using Equation 1.2, it is easy to get $\operatorname{Gut}\left(\Gamma_{n}\right)=(n-1)^{2}\left(W\left(\Gamma_{n}\right)\right)$.

(3) Combining with $K f^{*}\left(\Gamma_{n}\right)$ and $G u t\left(\Gamma_{n}\right)$, by using Theorem 3 (4), one gets

$$
\begin{gathered}
\lim _{n \rightarrow+\infty} \frac{K f^{*}\left(\Gamma_{n}\right)}{\operatorname{Gut}\left(\Gamma_{n}\right)}=\lim _{n \rightarrow+\infty} \frac{(n-1)^{2} K f\left(\Gamma_{n}\right)}{(n-1)^{2} W\left(\Gamma_{n}\right)} \\
=0
\end{gathered}
$$

This complete the proof of our theorem. 
Table 3.2: $K f^{*}(\Gamma)$ (reserves a decimal fraction) of graphs from $\Gamma_{4}$ to $\Gamma_{15}$.

\begin{tabular}{cccccccc}
\hline$\Gamma$ & $K f^{*}(\Gamma)$ & $\Gamma$ & $K f^{*}(\Gamma)$ & $\Gamma$ & $K f^{*}(\Gamma)$ & $\Gamma$ & $K f^{*}(\Gamma)$ \\
\hline$\Gamma_{4}$ & 174 & $\Gamma_{7}$ & 1078.8 & $\Gamma_{10}$ & 3370.5 & $\Gamma_{13}$ & 7696.4 \\
$\Gamma_{5}$ & 361.3 & $\Gamma_{8}$ & 1656.7 & $\Gamma_{11}$ & 4554.4 & $\Gamma_{14}$ & 9702.3 \\
$\Gamma_{6}$ & 655 & $\Gamma_{9}$ & 2412.6 & $\Gamma_{12}$ & 5988.4 & $\Gamma_{15}$ & 12030.3 \\
\hline
\end{tabular}

\section{Conclusion}

The graph spectrum has many useful information related to the graph. In this paper, we study the Kirchhoff, multiplicative Kirchhoff index and number of spanning trees for graph $\Gamma_{n}$. By using Decomposition theorem of Laplacian Polynomial, we procure that Laplacian spectrum of $\Gamma_{n}$ contains the Laplacian spectrum of complete graph $K_{n}$ and the Signless laplacian spectrum of the complete graph $K_{n}$. Further, the Gutman index and Wiener index for graph $\Gamma_{n}$ is also studied.

\section{References}

[1] E. Bendito, A. Carmona, A. M. Encinas, and J. M. Gesto, "A formula for the Kirchhoff index", International journal of quantum chemistry, vol. 108, no. 6, pp. 1200-1206, 2008, doi: 10.1002 /qua.21588

[2] M. Bianchi, A. Cornaro, J. L. Palacios, and A. Torriero, "Bounds for the Kirchhoff index via majorization techniques", Journal of mathematical chemistry, vol. 51, pp. 569-587, 2013, doi: 10.1007/s10910012-0103-x

[3] J. A. Bondy and U. S. R. Murty, Graph theory. New York, NY: Springer, 2008.

[4] F. Buckley and F. Harary, Distance in graphs. Redwood City, CA: Addison Wesley, 1990.

[5] A. Carmona, A. M. Encinas, and M. Mitjana, "Effective resistances for ladder-like chains", International journal of quantum chemistry, vol. 114, no. 24, pp. 1670-1677, Dec. 2014, doi: 10.1002 /qua. 24740 
[6] H. Y. Chen and F. J. Zhang, "Resistance distance and the normalized Laplacian spectrum", Discrete applied mathematics, vol. 155, no. 5, pp. 654-661, Mar. 2007, doi: 10.1016/j.dam.2006.09.008

[7] F. R. K. Chung, Spectral graph theory. Providence, RI: AMS, 1997.

[8] Z. Cinkir, "Effective resistances and Kirchhoff index of ladder graphs", Journal of mathematical chemistry, vol. 54, pp. 955-966, 2016, doi: 10.1007/s10910-016-0597-8

[9] G. P. Clemente and A. Cornaro, "Computing lower bounds for the Kirchhoff index via majorization techniques", MATCH communications in mathematical and in computer chemistry, vol. 73, n. 1, pp. 175-193, 2015. [On line]. Available: https://bit.ly/3eEWYfS

[10] G. P. Clemente and A. Cornaro, "New bounds for the sum of powers of the normalized Laplacian eigenvalues of graphs", Ars mathematica contemporanea, vol. 11, no. 2, pp. 403-413, 2016, doi: 10.26493/18553974.845.1b6

[11] I. Gutman, "Selected properties of the schultz molecular topological index", Journal of chemical information and computer sciences, vol. 34, no. 4, pp. 1087-1089, Sep. 1994, doi: 10.1021/ci00021a009

[12] I. Gutman and B. Mohar, "The quasi Wiener and the Kirchoff indices coincide", Journal of chemical information and computer sciences, vol. 36, no. 5, pp. 982-985, Sep. 1996, doi: 10.1021/ci960007t

[13] C. L. He, S. C. Li, W. J. Luo, and L. Q. Sun, "Calculating the normalized Laplacian spectrum and the number of spanning trees of linear pentagonal chains", Journal of computational and applied mathematics, vol. 344, pp. 381-393, Dec. 2018, doi: 10.1016/j.cam.2018.05.045

[14] J. Huang and S. C. Li, "On the normalized Laplacian spectrum, degreeKirchhoff index and spanning trees of graphs", Bulletin of the Australian Mathematical Society, vol. 91, no. 3, pp. 353-367, Jun. 2015, doi: $10.1017 / \mathrm{S} 0004972715000027$

[15] J. Huang, S. C. Li, and X. C. Li, "The normalized Laplacian, degreeKirchhoff index and spanning trees of the linear polyomino chains", Applied mathematics and computation, vol. 289, pp. 324-334, Oct. 2016, doi: 10.1016/j.amc.2016.05.024 
[16] D. J. Klein, I. Lukovits, and I. Gutman, "On the definition of the hyperwiener index for cyclecontaining structures", Journal of chemical information and computer sciences, vol. 35, no. 1, pp. 50-52, Jan. 1995, doi: 10.1021/ci00023a007

[17] D. J. Klein and M. Randić, "Resistance distance”, Journal of mathematical chemistry, vol. 12, pp. 81-95, Dec. 1993, doi: 10.1007/BF01164627

[18] J. B. Liu, J. Zhao and Z. X. Zhu, "On the number of spanning trees and normalized Laplacian of linear octagonal-quadrilateral networks", International journal of quantum chemistry, vol. 119, no. 17, Art ID. e25971, Sep. 2019. doi: 10.1002/qua.25971

[19] S. C. Li, W. Wei, and S. Q. Yu, "On normalized Laplacians, multiplicative degree-Kirchhoff indices, and spanning trees of the linear [n]phenylenes", International journal of quantum chemistry, vol. 19, no. 18, Art ID. e25863, Apr. 2019. doi: 10.1002/qua.25863

[20] J. L. Palacios, "Closed-form formulas for Kirchhoff index", International journal of quantum chemistry, vol. 81, no. 2, pp. 135-140, Nov. 2000, doi: 10.1002/1097-461X(2001)81:2<135::AID-QUA4>3.0.CO; $2-\mathrm{G}$

[21] Y. G. Pan and J. P. Li, "Kirchhoff index, multiplicative degree-Kirchhoff index and spanning trees of the linear crossed hexagonal chains", International journal of quantum chemistry, vol. 118, no. 24, Art ID. e25787, Dec. 2018, doi: 10.1002/qua.25787

[22] Y. G. Pan and J. P. Li, "Kirchhoff index, multiplicative degree-Kirchhoff index and spanning trees of the linear crossed polyomino chains", May 2019, arXiv:1905.06565

[23] Y. Peng and S. Li, "On the Kirchhoff index and the number of spanning trees of linear phenylenes", MATCH communications in mathematical and in computer chemistry, vol. 77, no. 3, pp. 765-780, 2017. [On line]. Available: https://bit.ly/2YIad9L

[24] H. Wiener, "Structural determination of paraffin boiling points", Journal of the American Chemical Society, vol. 69, no. 1, pp. 17-20, Jan. 1947, doi: 10.1021/ja01193a005 
[25] W. J. Xiao and I. Gutman, "Resistance distance and Laplacian spectrum", Theoretical chemistry accounts, vol. 110, pp. 284-289, Sep. 2003, doi: $10.1007 / \mathrm{s} 00214-003-0460-4$

[26] Y. J. Yang and H. P. Zhang, "Kirchhoff index of linear hexagonal chains", International journal of quantum chemistry, vol. 108, no. 3, pp. 503-512, 2008, doi: 10.1002/qua.21537

[27] H. P. Zhang, Y. J. Yang, and C. W. Li, "Kirchhoff index of composite graphs", Discrete applied mathematics, vol. 157, no. 13, pp. 2918-2927, Jul. 2009, doi: 10.1016/j.dam.2009.03.007

[28] H. Y. Zhu, D. J. Klein, and I. Lukovits, "Extensions of the Wiener number", Journal of chemical information and computer sciences, vol. 36, no. 3, pp. 420-428, May 1996, doi: 10.1021/ci950116s

[29] Z. Z. Zhu and J. B. Liu, "The normalized Laplacian, degree-Kirchhoff index and the spanning tree numbers of generalized phenylenes", Discrete applied mathematics, vol. 254, pp. 256-267, Feb. 2019, doi: 10.1016/j.dam.2018.06.026 